\title{
Lecturas ecológicas: hacia un canon de ecolecturas
}

\section{Reading ecology: towards a canon of ecoreadings}

\author{
Aitana Martos García ${ }^{1}$ \\ aitmartos@gmail.com \\ Universidad de Almería, España
}

\section{Resumen:}

Se defiende la necesidad de elaborar lecturas transversales con la educación medioambiental, literaria y del patrimonio, como ejes que sirvan para afrontar retos derivados de la crisis ambiental y de la pérdida de memoria cultural en los estudiantes y ciudadanos en general. Se examinan estas cuestiones a propósito de la cultura del agua como centro de interés, y se proponen criterios para la elaboración de un nuevo canon inclusivo, relativo a textos de literatura universal, con base en lecturas ecológicas. A tenor de estas prioridades, se describe la posible articulación de los ejes de conciencia ambiental, patrimonio cultural tangible e intangible, y la metodología didáctica más útil para promover lectores disidentes, que deseen poner en valor la cultura del agua propia de cada comunidad. Todo ello en consonancia con las nuevas corrientes educativas y culturales y en beneficio de la educación del ciudadano del siglo XXI.

\section{Palabras clave:}

Educación ambiental; educación literaria; patrimonio; cultura del agua.

\begin{abstract}
:
This article argues in favor of developing readings at the crossroads of environmental, literary and heritage educations. These will be the axis proposed to address challenges arising from the environmental crisis and the loss of cultural memory in students and citizens in general. These issues are explored especially in relation to the emblematic problem of water culture. We propose some criteria for the development of a new inclusive ecological canon with world literature texts. Based on these priorities, we describe the possible foundations of environmental awareness, of tangible and intangible cultural heritage and of the most useful teaching methods to promote dissident readers who wish to foster water culture within specific communities. All this is in keeping with new educational and cultural currents and seeks to have a positive impact on the education of $21^{\text {st }}$ century citizens.
\end{abstract}

\section{Key words:}

Ecoliteracy; literary education; heritage; water culture.

1 Dirección para correspondencia (correspondence address):

Aitana Martos García. Universidad de Almería. Departamento de Educación. Edificio

Científico Técnico III Matemáticas e Informática (CITE III). Almería (España). 


\begin{abstract}
Résumé:
Nous défendons la nécessité d'élaborer des lectures transversales entre l'éducation environnementale, littéraire et le patrimoine, qui sont des piliers servant à faire face aux défis découlant de la crise environnementale et de la perte de mémoire culturelle chez les étudiants et les citoyens en général. Ces questions sont examinées au regard de la culture de l'eau comme centre d'intérêt, et des critères sont proposés pour l'élaboration d'un nouveau canon inclusif, relatif aux textes de la littérature universelle, basé sur des lectures écologiques. Conformément à ces priorités, nous décrirons l'articulation possible entre la sensibilisation à l'environnement, le patrimoine culturel matériel et immatériel, et la méthodologie didactique la plus utile pour promouvoir les lecteurs dissidents qui souhaitent valoriser la culture de l'eau de chaque communauté. Tout cela dans la ligne des nouveaux courants éducatifs et culturels et au profit de l'éducation du citoyen du XXle siècle.
\end{abstract}

\title{
Mots clés:
}

L'éducation environnementale; éducation littéraire; patrimoine culturel; culture de l'eau.

Fecha de recepción: 18-09-2016

Fecha de aceptación: 11-09-2017

\section{Introducción: la ecoeducación y sus precursores. Modelos de educación ambiental.}

La ecoeducación se basa en el principio de la sostenibilidad y en las metodologías de la educación ambiental (Peliti, 2004). Estamos en un contexto innegable de crisis ambiental a una escala ya planetaria (Novo y Zaragoza, 2006), aunque se hace particularmente intensa en países mediterráneos como España, sometido a un estrés hídrico (Valladares et al., 2004) por la limitación y la irregularidad de las precipitaciones. Por eso, la "cultura del agua" es objeto de creciente atención, al evidenciarse las insuficiencias de los paradigmas dominantes. En todo caso, más allá de la gobernanza del agua, hace falta una visión holística de la cultura del agua que reintegre todos estos paradigmas (Martos y Martos, 2015), y que la perciba como un hecho cultural y no como una materia más de consumo.

Sin duda, los usos del agua forman parte del legado cultural, y son recogidos también en forma de "intangibles" o testimonios, incluyendo ahí los de carácter ficcional, a saber, los relatos, canciones, refranes, etc. de la tradición folclórica y literaria. Gracias al rechazo hacia la "voracidad" de los modelos de vida postindustriales y neocapitalistas, se ha extendido una corriente de libros defensores de una vuelta a la naturaleza e hipercríticos con la actual cultura industrial y urbana. 
El exponente esencial de esta tendencia es el filósofo y naturalista norteamericano Henry David Thoreau, quien defendía en Walden (Thoreau y Cramer, 1854) la vida sencilla en una cabaña a orillas del lago. Además de esta inmersión en el medio natural por más de dos años, a Thoreau se le conoce como adalid de la desobediencia civil, hasta el punto de que se le valora como un adelantado del preservacionismo actual y de los movimientos contestatarios anticapitalistas y ecologistas. El legado de Thoreau ha sido importante. La inmersión en el bosque venía a ser una aventura iniciática buscada -de ahí el imperativo de la soledad-, y para ello Thoreau se situaba en continuo diálogo vital con todo lo que le rodeaba, por tanto no era un retiro de renuncia al "mundo", sino de abrirse a "otro mundo más auténtico": los sonidos del bosque, sus quehaceres diarios, el cuidado de las plantas y cada una de las vivencias propias que nos cuenta con detalle sobre su vida en la cabaña del lago (Martos y Martos, 2016).

Así, desde una relectura actual de la experiencia de Thoreau, la única manera de abordar los graves problemas existentes de cara a las futuras generaciones es promover igualmente experiencias integradoras, que obliguen a pensar desde la complejidad (Morin y Pakman, 1994), pues la ecología es importante para reconocer estas tradiciones, pero también, y de forma fundamental, para vehicular una sensibilidad alternativa hacia el medio ambiente.

Y esto solo se puede lograr en el marco de la escuela. Por eso, como subraya Guimaraes (2004), toda educación es una educación ambiental, es decir, debe cimentarse en un diálogo continuo entre el entorno y la comunidad. Así, el medio ambiente no es un escenario o un decorado sino algo que compromete a la comunidad educativa, que debe promover el contacto con el entorno exterior, en todos sus sentidos, y construir una relación respetuosa y sostenible.

Al hilo de esto, Ferreira y Campina (2012) proponen tres modelos básicos de educación ambiental: conservacionista, pragmática y crítica, categorías que tratan de aunar multitud de tendencias, como la naturalista, conservacionista, solución de problemas, sistémica, holística, humanista, crítica, biorregional, feminista, entre otras (Sauvé, 2002). Desde el punto de vista de la lectura, es fácil integrar las tres perspectivas básicas, pues desde la lectura crítica, capaz de deconstruir estereotipos, es relativamente fácil obtener impulsos que modifiquen conductas en la línea de los conservacionistas y del tratamiento de problemas cercanos. 
Todo ello justifica aunar dimensiones como la conciencia ambiental, la educación literaria, el patrimonio cultural y la perspectiva de género, para afrontar los graves problemas derivados de la crisis social y ambiental, agravados en los últimos años. Tampoco es ajena a esto la pérdida de la memoria cultural en la escuela, pues la globalización, la tecnocracia y, en ciertos contextos, el desprecio de las culturas y tradiciones, han llevado a una rápida obsolescencia de muchas prácticas o rituales, asociados precisamente a la Naturaleza, como las Fiestas de Mayo u otras.

El paradigma de la Ecocrítica (Jamieson, 1996) supone un eje básico en este proceso, por cuanto genera un vuelco de dos preconcepciones asumidas como verdades en el pensamiento y la literatura occidental: la primera, que la Naturaleza es objeto (i.e. materia prima) y no sujeto, y la segunda, que el paisaje, por ejemplo, es un continente, esto es, un decorado, un escenario, y no un contenido o agente en sí mismo. El jardín japonés, por ejemplo, es una buena muestra de esta visión alternativa: tiene alma propia y está concebido para ser contemplado, no simplemente usado, y presta una atención especial a descubrir la singularidad del lugar (genius loci).

\section{Marco teórico: los imaginarios y el medio ambiente}

Los Imaginarios han sido objeto de un debate amplio, pero en todo caso nos interesan los imaginarios sociales (Castoriadis, 1999) como fuente de las representaciones más comunes, que afectan a nuestras percepciones sobre las artes, la moral, la economía, etc. En particular, la imaginación sobre el medio ambiente (Buell, 2001) es un ejemplo de los postulados de la ecocrítica (Jamieson, 1996), en cuanto a poner en valor el medio ambiente como eje que debiera impregnar la vida social. La estética, los imaginarios acerca del medio ambiente pueden vehicular contranarrativas (Barlow, 2008) que combaten los estereotipos más "tóxicos" que aún siguen modelando las conductas de los escolares.

Precisamente, la dicotomía entre "letras" y "ciencias" y la fragmentación de saberes explica la ausencia de Imaginarios que aparece en muchas prácticas de huertos ecológicos (Eugenio y Aragón, 2016), cuando es sabido que podemos aprender mucha ciencia a través de las narrativas (Negrete, 2002), por no hablar del interés que tienen los huertos como tópico literario (Sosa-Velasco, 2003). Hay, en efecto, numerosas 
leyendas sobre inundaciones, ahogamientos y otras catástrofes naturales, que son historias admonitorias y subrayan riesgos que entrañan la acción y la perspectiva andrópica, tal como vemos en las riadas causadas por una mala ocupación de los cauces de los ríos. Con una interpretación parabólica, al final, se convierten en tradiciones útiles porque hablan de la sostenibilidad, de la ética y la necesidad de respetar unos límites, en este caso, el cauce natural y las zonas inundables.

Al personificar en lamias, sirenas o encantadas, las leyendas también nos señalizan lugares de memoria (Nora, 2009), que la comunidad vincula a naufragios, ahogamientos $\mathrm{u}$ otros hechos atesorados en la memoria colectiva. Las distopías modernas (diluvios, catástrofes climáticas...) lo que hacen es amplificar este principio. Los Imaginarios del agua son un ejemplo de diversidad en todo el mundo, hasta el punto que se puede decir que cada comunidad y etapa tienen su propia cultura hídrica, asentada en un sistema de creencias y usos, y plasmada en un patrimonio tangible e intangible. Los imaginarios modernos del agua en la época moderna, desde el siglo XIX, no se vinculan ya a fuentes, cuevas o apariciones numénicas, sino a fenómenos emergentes como el senderismo, el montañismo, el deporte o el turismo. En efecto, el Ilamado "turismo de sol y playa" es lo más contrapuesto a las ninfas idílicas de la Égloga III de Garcilaso.

Se trata del tópico del locus amoenus, es decir, un paraje singular, silente, umbrío, solitario y rodeado de floresta, por tanto, no un lugar para bañarse, para ser "consumido" en "jolgorio", sino para admirar y contemplar. De hecho, la visión del baño de las doncellas está pautada en las culturas mediterráneas y tiene fuertes tabúes (recuérdese el episodio bíblico de Susana y los viejos, o el baño de la Cava, que está en el origen de pérdida de España).

En consecuencia, es el racionalismo moderno el que ha propiciado que ritos, creencias y "voces de la tierra" (Falas da terra, Guimaraes, 2004) se hayan vuelto más opacos, "invisibles" o irrelevantes a escala social. Es así como se ha generado esta reducción de la cultura del agua a la gestión hidráulica, entrando en obsolescencia la memoria cultural de las tradiciones del lugar, y con poca incidencia a la hora de la toma de decisiones sobre asuntos que conciernen a la ordenación territorial. Cada vez más estos espacios protegidos (De la Calle et al, 2006) se asocian a estrategias de sostenibilidad, incluyendo el valor de sus intangibles: es el caso del parque de Oromana, en Alcalá de Guadaira (Sevilla), 
que combina su legendario castillo almohade con un nuevo puente de cuño modernista (Puente del Dragón), visita a molinos harineros, acondicionamiento de la ribera, juegos educativos y cicloturismo (Rodríguez y Hermida, 2009).

Sin embargo, por citar un caso sintomático, aspectos como la hidrotoponimia (v.gr. Alcira, Guadix, etc.) son poco conocidos, y si se mencionan se hace como una rareza, curiosidad o información erudita. En cambio, es cierta la vinculación de la toponomia con ciertas leyendas fundacionales (Teruel, Toro, Torino...), de modo que el nombre remite a una especie de microrrelatos -formas condensadas de mitos o leyendas etiológicas-, que nos aportan una pista clara de un hecho relevante de la historia cultural de la comunidad y el lugar. En consecuencia, tales ecoficciones ayudan a visibilizar los citados lugares de memoria (Nora, 2009), y se plasman en formatos diversos (mitos, leyendas, rituales, símbolos en escudos municipales, etc.). Son Imaginarios que, a menudo, han sido fundacionales de paisajes culturales completos, como ocurre en el Camino de Santiago.

A fin de cuentas, el locus amoenus es un lugar de belleza y de ensimismamiento, como lo era la Sierra Morena para D. Quijote, al actuar conforme a los dictados de la novela pastoril. Pero en el contexto actual, el compartir cosas y estar conectados son valores de la posmodernidad y la era digital, por eso proponemos que la mirada poética se transforme también en una mirada crítica, para abordar algunas cuestiones centrales en nuestra historia cultural, donde medio ambiente, historia, economía o su reflejo en las artes están entrelazados.

Con todo, no se puede decir que los Imaginarios siempre provean de una representación idílica. Como expone Castoriadis (1999), se genera un "magma de significaciones" donde a veces también aparece lo más oscuro, gótico o siniestro. Se hace más necesario que nunca encauzar la mirada del estudiante y desarrollar sus facultades hacia una lectura crítica. El agua encantada es el agua apacible del locus amoenus -organizado en torno a una fuente o ribera-, pero también el agua desatada de la tempestad o simplemente el lugar que lleva a la muerte, como son todos los ríos del infierno en la imaginería de Dante, lugares poblados de monstruos y peligros. A veces, el mismo lugar que podría catalogarse como locus amoenus es en realidad un locus terribilis, como ocurre en la leyenda de Ojos verdes de Bécquer o en la historia clásica de Hylas, el amigo de Heracles desaparecido en una fuente. La mirada moderna 
de la ecocrítica trata de (re)conciliar estos contrarios, porque tanto la dimensión del agua que es luminosa y amable como la que es sombría y conlleva sufrimiento forman parte de la fenomenología de lo sagrado (Martos y Martos, 2016).

\section{La mirada poética, la mirada ecologista y la perspectiva de género}

El agua, desde las múltiples perspectivas humanísticas, como temática transversal, se configura como una de las áreas de interés en el campo de la Historia Cultural de un pueblo o región, uniendo la reflexión metodológica y teórica con la investigación empírica. En nuestro caso, entendemos la cultura del agua desde una triple perspectiva:

1. la basada en la operación de construcción de identidad, infiriendo el agua como elemento vertebrador de un pueblo;

2. la que considera el agua como elemento de la cultura patrimonial, dando lugar a múltiples textos escritos y textos transmitidos oralmente, que deben conservarse de forma organizada como potencial material educativo;

3. la que pretende entender el agua como forma patrimonial funcional, esto es, como una práctica encarnada en gestos, espacios y costumbres (la llamada cultura del agua), que forma parte de una memoria cultural viva.

Los imaginarios del agua nos llevan, en efecto, a una visión poliédrica del tema, incluyendo la posibilidad -por desgracia cercana- de un mundo sin agua, o con escasez, guerras u otros conflictos derivados de una ausencia de conciencia y sensibilidad ambiental. Es evidente, y está continuamente subrayado, que el desarrollismo a ultranza, el crecimiento sin tasa del parque de vehículos y la contaminación, el despilfarro en suma de los recursos naturales llevan a encrucijadas que solo con una clarividencia similar a la que tuvo Thoreau (1865) podremos resolver a largo plazo.

Entre tanto, la producción de Imaginarios se relaciona con la mirada poética, la cual fue definida por diversos formalistas como la desautomatización de la percepción (Ortí, 2004). En pocas palabras, no ver el bosque solo en términos de un depósito de maderas o un lugar de esparcimiento -esto es, en una visión andrópica- sino de forma más abierta, incluyendo la percepción de lo sagrado, de lo numénico (Otto, 2001). 
Debemos promover en todo caso la mirada propia del pensamiento lateral y creativo, la mirada "oblicua" que se desprende de los imaginarios del agua. Por citar un caso llamativo, la mayoría de los númenes o genios del agua son representaciones femeninas (Martos y Martos, 2013a). En efecto, las damas de agua son mucho más frecuentes que los otros dioses o monstruos marinos (Martos y Martos, 2015). Según Gimbutas (1989), las damas de agua, en toda su diversidad, son avatares de la Diosa Madre, configurando representaciones ligadas a la fertilidad, a la no violencia y a la cultura de la paz y la cooperación. Estos modos de vida de la Vieja Europa (Gimbutas, 1989) fueron sustituidos por el patriarcalismo, con sus carros de guerra, sus dioses olímpicos y su demonización de las mujeres (Ortíz-Osés, 1993).

Es así como las mujeres, ya desde los mitos griegos, se mudaron en furias, harpías, ménades o procreadoras de monstruos, aunque sin, a la vez, negarles su fuerza mágica, según se aprecia en el mito de Medusa y en su talismán, el gorgoneion, máximo objeto de poder en la cultura griega. Todo ello nos lleva a la perspectiva de género, pero nos enseña que cuando desde el Renacimiento el arte y la literatura occidental transmutaron estas damas de agua, por ejemplo, las ninfas y sirenas en seres lascivos, mitos eróticos o incluso demonios del mal para tentar al hombre, estaban haciendo una relectura interesada y moralista de estas ninfas de agua clásicas -genius loci o encantadas de las leyendas tradicionales-, apostadas en las riberas de los ríos o las grutas, y a quienes echaban ofrendas aquellos que buscaban tesoros y palacios encantados. A menudo, las ninfas no tienen ni cuerpo visible, son solo una voz, un rumor.

Del mismo modo, los dragones y otros monstruos devoradores (Martos y Martos, 2013b) han formado parte de estos Imaginarios del Agua, y en todos los casos la reacción de estos genios acuáticos dependía también de la pericia y astucia de los postulantes. Es algo que se ve en el cuento de los hermanos Grimm Frau Holle: dos niñas que acceden al ultramundo que hay al fondo del pozo, que no es ningún infierno dantesco ni un locus amoenus clásico, sino una representación ambiental ancestral propia de los indoeuropeos: un prado lleno de yerba. Dentro de él, tres tareas no menos significativas: hornear un pan, sacudir un manzano, voltear el edredón para que nieve sobre el mundo. El don o recompensa depende no tanto de que Frau Holle sea buena o malvada, sino según la pericia de los postulantes. De tal forma que los arquetipos 
se conservan siempre con matices, ya sea un cuento como este, o bien sagas o mundos narrativos completos, como Terramar, de U. Le Guin, o bien el océano pensante de Solaris, de S. Lem, y otras ficciones.

Lo importante, y de ahí la trascendencia de la educación lectora, es que el aprendiz no se quede con una lectura literal sin saborear los simbolismos que se van generando en la complejidad de crear un sentido. ¿Penélope es la mujer débil y fiel que espera a Ulises rodeada de pretendientes?, ¿o acaso es también la bruja del mar que ha aprendido el ardid de tejer y destejer el destino, como la araña, otra representación de la diosa madre? Lo que se entiende es que la educación ambiental y el feminismo tienen tales conexiones que coinciden en una misma filosofía: el ecofeminismo (Puleo, 2014).

Por tanto, la cultura del agua no es solo lo que se ve empíricamente, sino lo que ha sido imaginado, incluso cuando no hay agua, y ello es especialmente cierto cuando las tradiciones están llenas de historias de pozos y aguas subterráneas, o de aguas superiores, que descargan tormentas y tempestades.

En última instancia, las nuevas narrativas del agua (Barlow, 2008) preconizan principios tan simples como proclamar al agua como Bien Común, y no como una mercancía más, así como reforzar el principio democrático como mejor instrumento para proteger el agua a través de la conservación y la ley. Los ríos, por ejemplo, no conocen de estados, de fronteras y de otros imaginarios sociales (Castoriadis, 1999), pero en todos los testimonios culturales se aprecia siempre, ya sea de una cultura europea, semita o indoamericana, que un curso de agua natural es algo más que una simple conducción para riegos o desechos industriales, y que por eso desde la Antigüedad ya tenemos manifestaciones del tópico del locus amoenus, nucleado casi siempre en torno a una fuente o ribera y un paisaje frondoso que da sombra, es decir, árboles, hierba y agua en equilibro y armonía con el hombre.

\section{Criterios para una propuesta de intervención: patrones y ejemplos para el aula de Secundaria. De las prácticas a los textos, de lo local a lo universal}

Ya sabemos la complejidad del canon cuando hablamos de lecturas escolares (Mendoza, 2003), pero el tema es mucho más difícil si hablamos 
de lecturas ecológicas. Las antologías de ecolecturas suelen responder a criterios híbridos, es el caso de la Biblioteca de Villaviciosa de Odón², recogiendo libros expositivos, cuentos, manuales divulgativos, multimedias y obras centradas en temas como el reciclaje o la explicación de los ciclos naturales. Este criterio temático supuestamente centrado en la ecología, presenta a menudo contradicciones.

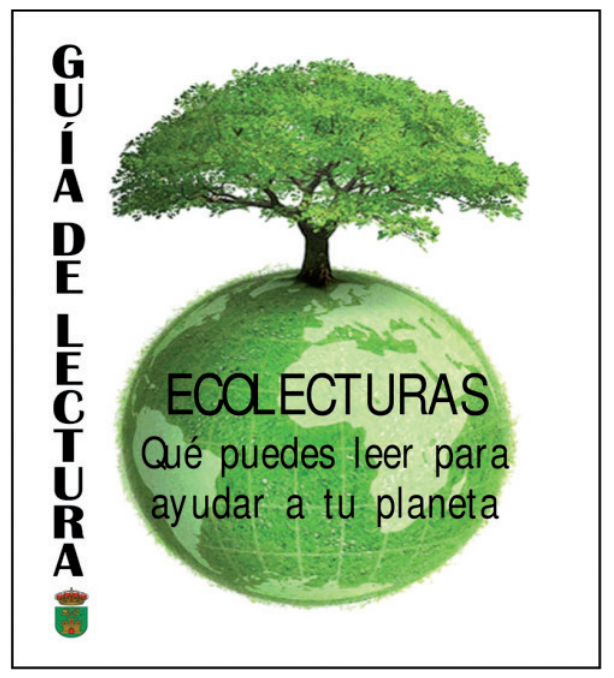

Por ejemplo, en este mismo catálogo no aparece la obra clásica de M. L. Gefaell, Las hadas de Villaviciosa de Odón, publicada en 1969. Ello denota un error grave a nuestro juicio: no primar lo que la Unesco llama la alfabetización situada, o dicho de otro modo, no incorporar todo el material patrimonial de los entornos de proximidad. De haberlo hecho así, el catálogo habría recogido la "Fuente de las Hadas", construida en el mismo pueblo, o hubiera documentado "pasajes" y "parajes" en homenaje a esta historia.

Acaso la razón está en que la biblioteca al hacer el catálogo etiqueta como ecológico ciertos estereotipos como los ya mencionados, y no repara lo suficiente en, por ejemplo, el vínculo de las hadas o las ninfas con las aguas. Así, el criterio de "proximidad" nos obligaría, como hiciera Pausanias al describir Grecia con un método corográfico, a buscar los lugares singulares, los monumentos, estatuas, historias (tradicionales y/o literarias), en fin, todo el patrimonio natural y cultural que atesora la

2 Véase una exposición detallada del catálogo de ecolecturas de la Biblioteca de Villaviciosa de Odón en https://issuu.com/bibliovillaviciosa/docs/ecolecturas 
memoria de esa comunidad. Sin ir más lejos, los árboles o bosques, los pozos, fuentes o riberas servirían de destino de estas posibles visitas o excursiones escolares. De modo que lo ecológico no está en predefinir una serie de items, como el reciclaje, sino en aplicar la "perspectiva ecocrítica" a cualquier lectura.

Nótese, pues, que no estamos aplicando un criterio centrado solo en los textos sino mucho más holístico e interdisciplinar. En esta fase, el profesor tiene que animar y organizar el proceso marcando uno de estos items, a través de una instrumentación didáctica como la que se adjunta ${ }^{3}$, basada en proponer una serie de itinerarios de lectura para trabajar con los textos tanto en el espacio convencional del aula como en salidas que constituyan una aproximación a un trabajo de campo. De hecho, el gran problema de la cultura del agua es su "invisibilidad", los alumnos no acceden a toda esa riqueza patrimonial de fuentes o pozos, y sus rituales asociados (v.gr. las ofrendas propias de los pozos de los deseos), porque su percepción está constreñida a la visión del agua como recurso "entubado", y a no ser conscientes de todo el valor socializador de esos

\section{EL ÁRBOL} SAGRADO

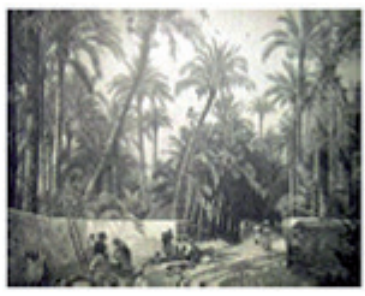

HERBOLARIOS Y ÁRBOLES

Leyendas Naturallistas enclaves, tal como hemos mostrado (Martos, 2015) a propósito de la fuente "La Cibeles" de Madrid. Valores que, por cierto, sí aparecen en los clásicos, como La Moza de Cántaro, de Lope de Vega, o en cuadros como Los aguadores, de Velázquez.

En esta misma línea de proyectos colaborativos que buscan la reconciliación entre los aspectos más instrumentales y los más emocionales, están también los llamados huertos literarios (Go-

3 Es la carátula y el índice de la Unidad didáctica "Árboles sagrados" para $3^{\circ}$ de la ESO del Proyecto "LECTURA Y PATRIMONIO CULTURAL DE ESPAÑA: LAS LEYENDAS TRADICIONALES COMO MATERIALES DE LECTURA.", elaborado por el Seminario de Investigaciones de Lectura para el Ministerio de Educación, Concurso Nacional de ayudas para la elaboración de materiales para facilitar la lectura en las diferentes áreas y materias del currículo (BOE-18-IX-2007). Proyecto concedido en BOE 19-III. 2998, p. 1669. Mat. inéd. 
zalbo y Aragón, 2016), que buscan lo inverso: reconvertir el espacio del aula en espacio cuasi natural, donde las flores brotan en forma de dibujos y poemas, aunque, como ocurre con el jardín japonés, requiere de un trabajo previo de observación y concentración.

Desde la perspectiva de los textos, los enfoques son también amplios, podemos tomar estos mismos temas, el árbol, el pozo o el pez (Bordons y Díaz Plaja, 2008). En cuanto a los textos tradicionales, proponemos, pues, ir desde el lugar o la Naturaleza próxima a los textos, con énfasis, claro está, en las leyendas tradicionales y otras clases de narrativas míticas. De este modo integraríamos historia, paisaje y textos. Es algo que también podemos hacer igual a propósito de los textos literarios. No se trata de seguir al pie de la letra las correspondencias entre el autor o la obra y la ciudad, pero sí de indagar las afinidades.

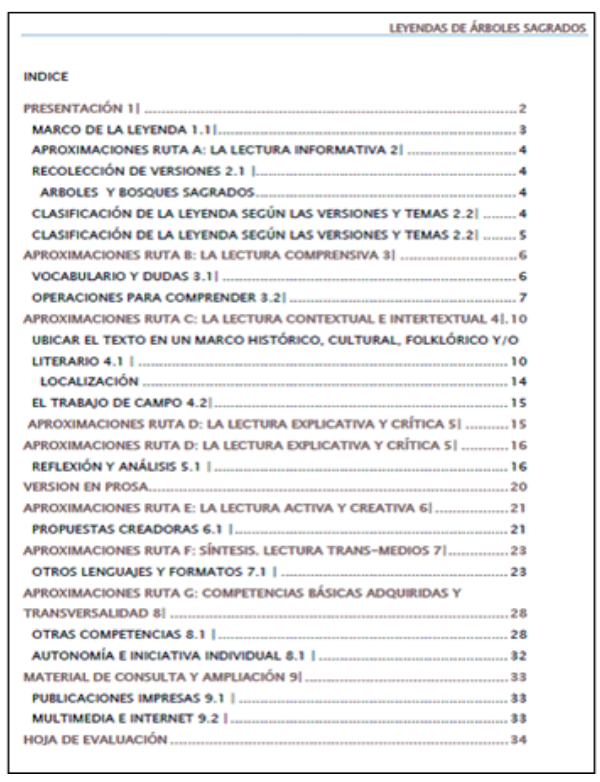

Dicho de otro modo, si el ambiente de Sevilla encaja con el romanticismo de Bécquer, o los campos sorianos con A. Machado, o la Mancha con el Quijote, cabe explorar esta visión, y hacer rutas o actividades externas integradas con la programación de la materia. Rebasando ya el ámbito de lo local, las tradiciones de otros pueblos y la literatura infantil/ juvenil son "viveros" de ecolecturas por su conexión con el niño y las representaciones del medio ambiente (Harding, Waller y Thiel, 2009).

A este respecto, dicen estos autores que tenemos numerosos textos 
clásicos de diversas culturas y de la literatura universal, porque sería absurdo parcelar la conciencia ambiental por lenguas, países o regiones; a modo solo de muestra, cabría citar el clásico de Kingsley The Water Babies (1863); The Pool of Tears (Lewis Carroll, Alicia en el país de las maravillas, 1865); Louisa May Alcott, Ripple, the Water-Spirit (Flor de Fábulas, 1855); el cuento budista The Princess and the Water-Sprite (Jataka, siglo III a.C.); la leyenda siciliana de Colapesce; Edith Nesbit, la inspiradora de Lewis y Rowling, en su obra Wet Magic (1913); O. Preussler, The Water Sprite (2001); A. Bouchard, Acqua dolce (2008), A Menina do Mar (1958) de Sophia de Mello Breyner, y otras obras similares, sin olvidar la literatura infantil y juvenil española, como Las raíces del mar, de Fernando Alonso, basada en la leyenda de la isla de Ys, o La huida del mar, de Agustín Fernández Paz, y la saga Relatos de Mirthad (con su primera entrega, Las Tierras en Juego, 2015) de León Muela, que contiene una declaración explícita de principios:

"Los ríos, bosques, montañas y tierras en general no eran propiedad de nadie, por lo que los elfos creían que los humanos no tenían derecho a contaminar el río Basol. [...] Estamos hablando de la poca concienciación de los humanos por el respeto a la naturaleza" (2015:5).

No citamos aquí las diversas películas de animación de Disney, Pixar, etc. que también recrean mundos marinos, a menudo con una mirada amable y colorista, por tanto edulcorada y lejana a las demandas que estamos examinando. Algo similar cabe decir de otras producciones multimedia, anime, cómic... pues todas muestran un paisaje que es a menudo una caricatura del locus amoenus o, en el caso de las distopías, de sus antípodas, el locus terribilis, con una escasa comprensión de los símbolos en juego, lo cual lleva a estereotipos que desde luego no contribuyen a la visión holística que preconizamos. En cambio, filmes como Cuando el destino nos alcance (Soylent Green), versión dirigida por R. Fleischer en 1973 sobre la novela ¡Hagan sitio! de 1966 de Harry Harrison, suponen un crudo alegato contra la superpoblación, la corrupción, la manipulación o las drogas como horizontes distópicos de vida. Por su parte, la literatura ha producido obras recientes muy reveladoras a este respecto, como Algo, ahí fuera, novela publicada por Bruno Arpaia en 2016 o Dos mil noventa y seis, de Ginés Sánchez, publicada en 2017, textos 
ambos que ponen el énfasis en los temas aludidos de la superpoblación, el cambio climático, etc.

En síntesis, remover la conciencia de los valores ambientales, patrimoniales y literarios en los alumnos debería llevarnos al inconformismo, como en Thoreau, al poner en valor el medio ambiente, la herencia cultural o la lectura. Walden no es un libro místico o sentimental al modo romántico, sino bastante pragmático, pues se centra en las necesidades de cada día, pero a la vez espiritualista, porque al fin y al cabo lo que se busca es una comunión con la naturaleza. En esa medida, es un proceso también ascético, de desligarse de todo lo que -como la prisa- entorpece esta comunicación. Hoy en día, la adicción por las pantallas, el (ab)uso de las redes sociales o las modas "meméticas" (como la pokemanía de los móviles), recomendarían, precisamente, una vuelta a estos valores básicos, y de hecho, se han llevado a experiencias scouts para senderismo y "ayuno de pantallas", como una suerte de escapadas o estancias iniciáticas al modo de Thoreau. Recuperar la infancia (Savater, 1994) es también recuperar estas experiencias y conflictos primarios, como la amistad, la experiencia del mar o la rebeldía contra las injusticias.

\section{Conclusiones: un enfoque holístico para impulsar la ecoliteracidad en nuestros alumnos}

En conclusión, los problemas ambientales no solo deben contemplarse desde la perspectiva tecnológica o de las ciencias experimentales, sino que requieren una visión holística y crítica. No puede reducirse la educación medioambiental que se centra en los tópicos del catálogo que citábamos de la Biblioteca Ecológica de Villaviciosa de Odón. Nos parece importante, educativamente hablando, la narratividad, los imaginarios y el ficcionalizar estas tradiciones para entender estas aguas no como una masa homogénea, sino como aguas "heterogéneas", es decir, singulares, con nombres y alma propia (de ahí las leyendas de xanas y otras figuras asociadas a esos enclaves como "genius loci"). Todo ello desde una mirada reflexiva, crítica, que se "deleite" en estos temas y que los aprecie emocionalmente (Honoré, 2007).

Pero las lecturas ecológicas, a través de la recuperación de mitos, cuentos, canciones, libros de literatura infantil o clásicos leídos en clave ecocrítica, no solo ayudan a forjar esta conciencia ecológica. Tienen 
otras conexiones con la salud, el deporte o el desarrollo local, al promover por ejemplo el senderismo y recorrer con ayuda de rutas o itinerarios algunos "lugares de memoria" del entorno, vinculados a tradiciones locales. Las lecturas ecológicas tienen otras importantes connotaciones: educación del consumidor, por lo que supone de despertar en los ciudadanos un interés crítico hacia los temas alimentarios; perspectiva de género, en conexión con el ecofeminismo, porque hacer visible la naturaleza es también visibilizar el papel de las mujeres en muchos ámbitos. Por añadidura, la comprensión intercultural, porque, por ejemplo, al entender la heterogeneidad de usos y rituales referidos al agua (baños, abluciones, etc.), se aprende a respetar las diferentes culturas.

Con todo, según ya apuntamos, lo ecológico no está tanto en el tema como en la perspectiva crítica. De hecho, rescatar la mirada crítica propia del ecofeminismo o de la ecoetnología supone saber incorporar y respetar la perspectiva de la naturaleza, de la mujer o de la sabiduría ancestral, lo cual es un buen camino hacia una alfabetización ciudadana. A tal fin, urge recuperar del patrimonio oral las ecoficciones, en forma de leyendas, canciones o cuentos sobre historias de la naturaleza, así como releer a los clásicos en clave ecocrítica, como la novela pastoril, el cancionero tradicional, los relatos costumbristas o los libros de viajes.

La cultura del agua es un buen ejemplo de ello. De hecho, el ecologismo ha sido incorporado a menudo más como una moda que como una realidad que ponga en valor la herencia cultural y el papel de la lectura como modeladora de valores. La saga Avatar es un ejemplo de una obra de éxito que promueve valores alternativos susceptibles de provocar una comprensión global de los retos futuros. Por el contrario, la fragmentación de estos movimientos alternativos ha perdido el sentido holístico del naturismo del siglo XIX, para desgajarse en multitud de subtendencias -vegeterianos, veganos, animalistas...- que inciden en distintos problemas o áreas de contenidos, como las dietas, las plantas o el posicionamiento ante temas conflictivos en la opinión pública: por ejemplo, los parajes protegidos, los trasvases, el trazado de autopistas, la tauromaquia, el reciclaje..., pero todo ello de forma muy atomizada y por tanto difícil de instrumentar en educación.

En todo caso, la obra y el ejemplo de Thoreau demuestran que no se pueden separar los problemas y valores políticos y ambientales, de modo que el movimiento pro derechos civiles se alinea con el movimiento ambiental, al igual que el feminismo y la ecocrítica se hermanan 
al buscar visibilizar y empoderar a entidades, para así darles voz, las falas da terra (Guimaraes, 2004).

Las decisiones ambientales son también, pues, opciones de valores en el aula, y por eso debemos restablecer las conexiones entre el canon de lecturas en el aula, los métodos de enseñanza y las demandas/compromisos éticos más urgentes del mundo actual, a fin de evitar la depredación de la Naturaleza o los abusos hacia la mujer y otros colectivos, al igual que Thoreau en su tiempo se comprometió contra el esclavismo.

En suma, los Imaginarios del agua no son solamente fabulaciones. La estética y las representaciones acerca del medio ambiente pueden vehicular contranarrativas del paisaje (Duarte, 2010), que compensen estereotipos y prejuicios que han modelado las creencias y conductas de los escolares, como el consumismo o el culto al desarrollismo y la tecnología sin más. Para todo ello, necesitamos recuperar ecoficciones y promover un canon inclusivo y transversal, capaz de reutilizar los fenómenos modernos (turismo, robótica, deporte) conciliándolos con nuestra herencia cultural, pero que a la vez no rehúya los temas candentes, como el cambio climático o la superpoblación.. También deberíamos integrar todos los géneros, ficcionales y no ficcionales, con textos tan señalados como el citado Walden de Thoreau, que no es un texto de ficción sino un libro de memorias e impresiones, y por ello algo cercano a una historia de vida: La llamada de la selva, de Jack London; El mundo del fin del mundo, de Luis Sepúlveda; En nombre de los mares, de Paul Watson; El sabor de Fukushima, de Ryoko Sekigushi, y muchos más.

Es cierta, a nuestro juicio, la intuición de Thoreau: el ecocentrismo es una alternativa real al posthumanismo en que ya estamos instalados, una vez que el avance científico permite que la humanidad se "rediseñe", por así decir, pero donde, a su vez, estamos a las puertas mismas de agotar o contaminar los recursos naturales básicos, y debe conciliarse con el humanismo y la tecnología.

No es fácil responder a todas las demandas, retos y controversias que afectan en su mayoría al tipo de sociedad y naturaleza que deseamos. En todo caso, el enfoque defendido demuestra que la exaltación de la naturaleza no es simplemente una opción pedagógica, sino la vía necesaria para crear esa conexión entre las diversas dimensiones de la vida, entre lo natural, lo social y lo espiritual. Sin una ética y una estética del paisaje solo sabremos sufrir una patética, esto es, las distopías, catástrofes y conflictos por llegar, sequías, guerras y usos inapropiados e insolidarios 
del medio ambiente. La educación integrada que proponemos para desarrollar la ecoliteracidad y la interculturalidad (Morgado y Pires, 2010) no solo aspira a interrelacionar competencias básicas, se dirige al núcleo mismo que animó a Thoreau y hoy moviliza a miles de turistas: se trata del aprendizaje como experiencia, de experimentar la naturaleza, el patrimonio, las lecturas, con otros ojos y desde otros posicionamientos, que un aula más heurística e indagadora ayude a descubrir.

Tampoco es conveniente frivolizar a la hora de ponderar las metodologías que necesitamos: las acampadas, las rutas o las "quedadas" sirven de poco si es para cazar pokemons. Necesitamos articular proyectos sólidos y ambiciosos que pongan en valor, por ejemplo, la cultura del agua, y para ello las TIC serán siempre buenos aliados, pero debemos trazar itinerarios y programas consistentes. Por ejemplo, el citado autor Arkaitz León (2015) basaba su saga en su propia experiencia de ecología social y vida comunitaria en Lakabe, un pueblo de Navarra. La autenticidad ecologista de muchos de sus planteamientos recuerda, pues, a la de Thoreau, solo que en este caso se echa mano de la ficción para hablar de la realidad.

\section{Referencias}

Barlow, M. (2008). Our Water Commons: Toward a new freshwater narrative. Ottawa: Council of Canadians.

Bordons, G. y Díaz Plaja A. (2008). Peces. Un tema universal como pasarela entre diversas literaturas. Lenguaje y Textos, (28), 43-74.

Buell, L. (2001). Writing for an Endangered World: Literature, Culture, and Environment in the U.S. and Beyond. Cambridge, MA and London: The Belknap Press of Harvard University Press.

Cartea, P. A. M. (2006). Crisis ambiental y globalización: Una lectura para educadores ambientales en un mundo insostenible. Trayectorias: revista de ciencias sociales de la Universidad Nacional de Nuevo León, (20), 110-123.

Castoriadis, C. (1999). L'institution imaginaire de la société. Paris: Editions du Seuil.

De La Calle Vaquero et al. (2005). Los espacios protegidos en España: significación e incidencia socioterritorial. Boletín de la Asociación de geógrafos Españoles, (39), 227-266.

Duarte, M. (2010). Contranarrativas del paisaje en cuatro actos: Magda Portal, Pedro Nel Gomez, Fernando Vallejo y Blanca Wiethiichter. Pittsburgh: Monasterios.

Eugenio Gozalbo, M. y Aragón Núñez, L. (2016). Experiencias en torno al huerto ecológico como recurso didáctico y contexto de aprendizaje en la formación inicial de maestros de Infantil. Eureka sobre enseñanza y Divulgación de las Ciencias, 13(3), 667-679. 
Lecturas ecológicas: hacia un canon de ecolecturas

Fernández Rodríguez N. (2014). El valor estructural de la magia en el universo pastoril de Lope de Vega: convención, vitalismo y parodia. EHumanista, (26), 1-17.

Ferreira Da Silva, R. L., y Campina, N. N. (2012). Concepções de educação ambiental na mídia e em práticas escolares: contribuições de uma tipologia. Pesquisa em Educação Ambiental, 6(1), 29-46.

García De Diego, V. (1958). Antología de leyendas de la literatura universal, 2 vols. Labor. Barcelona.

Gimbutas, M. A. (1989). The language of the goddess: Unearthing the hidden symbols of western civilization. San Francisco: Harper \& Row.

Gozalbo, M. E. y Aragón Núñez, L. (2016). Experiencias en torno al huerto ecológico como recurso didáctico y contexto de aprendizaje en la formación inicial de maestros de Infantil. Revista Eureka sobre Enseñanza y Divulgación de las Ciencias, 13(3). 667-679.

Guimaraes, A.P. (2004). Falas da Terra - Natureza e Ambiente na tradição popular portuguesa. Lisboa: Colibrí.

Harding, J., Waller, A., y Thiel, E. (Eds.). (2009). Deep Into Nature: Ecology, Environment and Children's Literature. Lichfield: Pied Piper Publishing.

Honoré, C. (2007). Elogio de la lentitud. Madrid: RBA Coleccionables.

Jamieson, D. (1996): The Ecocriticism Reader: Landmarks in Literary Ecology (org. Cheryll Glotfelty e Harold Fromm). Georgia: University of Georgia Press.

León Muela, A. (2015). Las Tierras en Juego. Navarra: Círculo Rojo.

Martos García, A. E. y Martos García, A. (2015a). Poética del agua en las narraciones tradicionales textos y contextos: texts and contexts. Literatura y lingüística, (32), 41-62.

Martos García, A.E. y Martos García, A. (2015b). Nuevas lecturas de La Llorona: imaginarios, identidad y discurso parabólico. Universum (Talca), 30(2), 179-195.

Martos Núñez, E. y Martos García, A. E. (2012). Los imaginarios del agua y sus lecturas pansemióticas. Álabe, (6).

Martos Núñez, E. y Martos García, A. (2013a). Ecoficciones e imaginarios del agua y su importancia para la memoria cultural y la sostenibilidad. Alpha (Osorno), (36), 71-91.

Martos Núñez, E. y Martos García, A. E. (2013b). Imaginarios del devoramiento en la cultura del agua: dragones," tragantía", tragaldabas y otros espantos. Indivisa: Boletín de estudios e investigación, (13), 122-143.

Martos Núñez, E. y Martos García, A. (2015). Memorias e imaginarios del agua: nuevas corrientes y perspectivas. Agua y Territorio, (5), 121-131.

Martos Núñez, E. y Martos García, A. (2016). Ecoeducación y Cultura del Agua, un reto para la escuela del s.XXI. Educación Permanente. Homenaggio a Lanfranco Rosati. Roma: Giufré Editore

Mendoza (2003). El canon formativo y la educación lecto-literaria. Mendoza (Coord.), Didáctica de la Lengua y la Literatura, Pearson Educacion, Madrid, 349-379.

Morgado, M. y. Pires, N. (2010). Literatura Infantil e Educação Intercultural. Vivemos num Mundo sem Esconderijos. Lisboa: Colibrí.

Morin, E., y Pakman, M. (1994). Introducción al pensamiento complejo. Barcelona: Gedisa. 
Negrete, A. (2002). Science Via Narratives Communicating Science through Literary Forms. Ludus vitalis, 18, 197-204.

Nora, P. (2009). Los lugares de la memoria. Santiago: LOM.

Novo, M., y Zaragoza, F. M. (2006). El desarrollo sostenible: su dimensión ambiental y educativa. Madrid: Pearson.

Ortí, P. S. (2004). Desautomatización y creación. Dicenda: Cuadernos de filología hispánica, (22), 249-269.

Ortiz-Osés, A. (1993). Las claves simbólicas de nuestra cultura: matriarcalismo, patriarcalismo, fratriarcalismo (Vol. 13). Madrid: Anthropos Editorial.

Otto, R. (2001). Lo racional y lo irracional en la idea de Dios. Madrid: Alianza EditorialEmecé.

Peliti, M. (2004). Dall'educazione ambientale all'educazione sostenibile: I'Eco-School. Tesis Doctoral. Universidad de Ferrara.

Puleo, A. H. (2014). Ecofeminismo para otro mundo posible. Madrid: Ediciones Cátedra.

Redondo-Olmedilla, J. C. (2015). A Companion to Australian Aboriginal Literature edited by Belinda Wheeler. The Goose, 14(1). Art. 39. http://scholars.wlu.ca/thegoose/ vol14/iss1/39

Rodríguez Freire, J., y Hermida Ruiz, J. (2009). Alberinto. Juego de enigmas, acción y aventuras en ocho episodios sobre la historia y el patrimonio en Alcalá de Guadaíra ( $\left.N^{\circ} 15\right)$. Madrid: Ministerio de Educación.

Sauvé, L (2002). Environmental Educations: possibilities and contraints. Connect, v. XXVII, 1-4.

Savater, F. (1994). La infancia recuperada. Madrid: Taurus.

Sosa-Velasco, A. J. (2003). El huerto de Melibea: Parodia y subversión de un topos medieval. Celestinesca, 27(1), 125-148.

Thoreau, H. D., y Cramer, J. S. (1854) Walden. London: Yale University Press, edición de 2006.

Valladares, F., Vilagrosa, A., Peñuelas, J., Ogaya, R., Camarero, J. J., Corcuera, L. y Gil-Pelegrín, E. (2004). Ecología del bosque mediterráneo en un mundo cambiante, Estrés hídrico: ecofisiología y escalas de la sequía 2. Madrid: Ministerio de Medio Ambiente, 165-192. 
